\title{
Efecto de un programa de educación física regular y cuentos motores en la aptitud física y desarrollo motor de escolares costarricenses
}

\section{Effect of a physical education regular program and motor stories in the physical suitability and motor development of costarricane schools}

\author{
M. Sánchez-Méndez, A. Salicetti-Fonseca y J. Hernández-Elizondo
}

Facultad de Educación, Escuela de Educación Física y Deportes, Universidad de Costa Rica, San José, Costa Rica.

\begin{abstract}
Resumen: Se determinaron cambios en la Resistencia Cardiovascular [RCV], Fuerza del Extensor del Tronco [FET] y Destrezas Fundamentales de Movimiento [DFM] en niños, luego de recibir un Programa de Educación Física Regular [PEFR]; o, un Programa de Cuentos Motores [PCM]; y, comprobando diferencias entre Actividad Física Moderada y Vigorosa [AFMV]. 55 estudiantes completaron el estudio, distribuidos en tres grupos PEFR, PCM y Grupo Control (GC). Los grupos recibieron 20 clases no el GC. Se contabilizó el tiempo de AFMV mediante $P O L A R^{\circledR}$. Se utilizó FITNESSGRAM ${ }^{\circ}$ y $T G M D-2^{\circ}$ para medir variables. La RCV y FET en grupos intervenidos mejoró. Las DFM en ambos grupos intervenidos mejoró. A pesar de que los sujetos mantuvieron un Coeficiente de Desarrollo Motor Grueso (CDMG) bajo; los programas coadyuvaron a su mejoría, no así el GC que conservó un CDMG muy pobre. El PCM y el PEFR fueron eficaces para generar cambios en aptitud física y destrezas analizadas.

Palabras clave: salud escolar, intervención educativa, FITNESSGRAM ${ }^{\odot}$, desempeño motor
\end{abstract}

Abstract: Changes in Cardiovascular Resistance [RCV], Trunk Expander Force [FET] and Fundamental Movement Skills [DFM] in children were determined after receiving a Regular Physical Education Program [PEFR]; or, a Motor Story Program [PCM]; and, verifying differences between Moderate and Vigorous Physical Activity [AFMV]. 55 students completed the study, divided into three groups PEFR, PCM and Control Group (GC). The groups received 20 classes, not the GC. AFMV time was accounted for using POLAR ${ }^{\oplus}$. FITNESSGRAM ${ }^{\circledR}$ and TGMD- $2^{\circledR}$ were used to measure variables. The RCV and FET in operated groups improved. The DFM in both intervention groups improved. Although the subjects maintained a Low Coefficient of Motor Development (CDMG); the programs contributed to its improvement, but not the GC that kept a very poor CDMG. The PCM and the PEFR were effective in generating changes in physical fitness and skills analyzed.

Keywords: school health, educational intervention, FITNESSGRAM ${ }^{\odot}$, motor performance.

\section{Introducción}

Para alcanzar y mantener un peso saludable en escolares, el Colegio Americano de Medicina Deportiva (ACSM, 2018), promueve directrices como: (1) alcanzar diariamente al menos ochenta minutos de Actividad Física (AF) de cualquier intensidad en adición a los sesenta minutos mínimos de Actividad Física Moderada y Vigorosa (AFMV) diaria, (2) hacer AF para la activación de grandes grupos musculares, (3) alcanzar un adecuado desarrollo motor grueso y aptitud física; y (4) abstenerse de pasar inactivos por más de sesenta minutos en cada conducta sedentaria, exceptuando las horas del sueño. Además, insta a las autoridades la inclusión de la asignatura de Educación Física en todas las instituciones escolares, caracterizada por un programa de condición física que desarrolle la aptitud física del alumnado, especialmente la resistencia aeróbica, músculo esquelética, el desarrollo motor grueso y aporte una cantidad de AFMV mínima del 50\%

Dirección para correspondencia [Correspodence address]: Alejandro Salicetti-Fonseca. Facultad de Educación, Escuela de Educación Física y Deportes, Universidad de Costa Rica, San José (Costa Rica). E-mail: alejandro.salicetti@ucr.ac.cr del tiempo total dedicado AF en las lecciones. Escriche, $\mathrm{Mu}-$ ńoz, Sos, \& Casterad, (2018).

Por consiguiente, el Ministerio de Educación Pública costarricense (MEP) insta la ejecución del eje transversal de "Educación para la Salud" con el propósito de promover estilos de vida saludable en las instituciones escolares (ArceLeón \& Claramunt-Garro, 2009). En este sentido, las clases de Educación Física son un factor clave para que el alumnado se ejercite, realice AFMV e identifique hábitos inadecuados para la salud y busque modificarlos.

Las intervenciones educativas en Educación Física diseñadas específicamente para generar cambios en componentes de aptitud física y motriz en niños escolares, obtienen resultados significativos (Morgan et al., 2013; Eather et al., 2013; Logan, Webster, Getchell, Pfieiffer, \& Robinson, 2015) Asimismo, se tiene conocimiento sobre la eficacia de intervenciones educativas para aumentar la cantidad de AFMV en las escuelas (Sbruzzi et al., 2013). Sin embargo, se sabe muy poco sobre la eficacia de las intervenciones educativas que utilizan Cuentos Motores. Las investigaciones en el área son relativamente recientes y muy escasas. Además, han utilizado únicamente métodos de investigación de corte cualitativo, en cuyo caso, 
se ha encontrado que aplicar Cuentos Motores en las clases de Educación Física favorece el desarrollo del esquema corporal, el aprendizaje de destrezas motrices básicas y el disfrute de la AF en el medio natural (Herrera \& Barbero, 2013; Reyes Alonso, 2013; Aparicio Sacristán, 2014). Hasta el momento, no se han encontrado estudios publicados sobre el efecto de un Programa de Cuentos Motores en variables de aptitud física y desarrollo motor grueso en niños y niñas escolares.

Este estudio surge de la necesidad de generar mayor investigación sobre estrategias de Educación Física eficaces para: fortalecer acciones de promoción de la salud en el ámbito escolar (Ministerio de Salud Costarricense, 2009) y tuvo como objetivo comparar el efecto de dos programas de Educación Física sobre variables de aptitud física, AFMV y desarrollo motor grueso en escolares que no contaban con lecciones o profesorado de Educación Física en la institución.

\section{Metodología}

Esta investigación se desarrolló en dos instituciones educativas costarricenses, que no contaban con lecciones, ni profesorado de Educación Física. El reclutamiento consideró la participación autorizada de los nińos y nińas una vez que los padres de familia firmaron la fórmula del Consentimiento Informado que describe el objetivo, protocolo, riesgos y beneficios del estudio, avalado por el Comité Ético Científico de la Universidad de Costa Rica (VI-3873-2015).

\section{Procedimientos de medición}

Al inicio del estudio, se medió el peso corporal, la talla y se calculó el Índice de Masa Corporal (IMC) de los sujetos con fines descriptivos. En dos convocatorias, antes y después de aplicar los programas de estudio, se midieron dos componentes de aptitud física: Resistencia Cardiovascular (RCV) y Fuerza del Extensor del Tronco (FET), utilizando la batería de pruebas FITNESSGRAM ${ }^{\oplus}$ (2014), basada en las guías estadounidenses válidas para cuantificar componentes de aptitud física de niños y nińas en estudios a nivel de campo.

Se eligió la prueba de Course Navette para medir la RCV de los sujetos en $\mathrm{VO}_{2}$ máx., estimado $\left(\mathrm{ml} \bullet \mathrm{kg}^{-1} \bullet \mathrm{min}^{-1}\right)$. Los sujetos recorrieron una distancia de 20 metros delimitada con líneas en el piso (ida y vuelta) de manera ininterrumpida al ritmo que marcó un pitido de la grabación según el protocolo de la prueba. La cual finalizó cuando el ejecutante no logró pisar la línea en dos ocasiones consecutivas al momento de la señal correspondiente. Los valores de $\mathrm{VO}_{2}$ máx., fueron calculados mediante la siguiente ecuación:

$\mathrm{VO}_{2}$ máx. $=31.025+3.238 \mathrm{~V}-3.248 \mathrm{E}+0.1536 \times \mathrm{E} \times \mathrm{V}$
En la que "V" es la velocidad que corresponde a la última vuelta completada y "E" la edad del sujeto (García \& Secchi, 2014). El resultado obtenido se clasificó en un rango de aptitud física saludable según la tabla del valores del FITNESSGRAM .

Por otra parte, se eligió la prueba Trunk Extensor Strength - Trunk; FITNESSGRAM ${ }^{\circledast}$ (2014), para medir la FET de los sujetos en centímetros $(\mathrm{cm})$. El sujeto se colocó de cúbito ventral sobre una colchoneta colocada en el piso, y ejecutó una extensión del tronco sostenida por 5 segundos, ambas extremidades superiores pegadas al torso y la cabeza recta con mirada hacia el frente para proceder a la medición de la distancia existente entre la barbilla del sujeto y la colchoneta. La medición indicó un grado de fuerza isométrica expresada en $\mathrm{cm}$. El valor de medición obtenido se clasificó según una tabla de puntuaciones estándar para valorar su fuerza en un rango de aptitud física saludable.

Así también, antes y después de aplicar los programas de estudio, se midió el Coeficiente del Desarrollo Motor Grueso (CDMG) a partir de la evaluación de doce Destrezas Fundamentales de Movimiento (DFM): seis Movimientos de Locomoción (ML): carrera, galope, deslizamiento, zancada, brinco y salto horizontal. Y seis movimientos que implican Control del Objetos (CO): apañar, rebotar, lanzar sobre hombro, lanzar por debajo de hombro, patear y batear. Los sujetos ejecutaron en dos intentos cada una de las doce DFM aplicadas, según el protocolo de la prueba de Desarrollo Motor Grueso (TGMD-2 ${ }^{\circledR}$ ), de Ulrich (2000). La prueba implicó obligatoriamente la grabación de cada una de las ejecuciones para su posterior análisis.

Finalmente, se registró el tiempo efectivo (minutos) en que los participantes realizaron AFMV durante los programas, utilizando pulsómetros $P O L A R^{\oplus}$, para la monitorización de la frecuencia cardíaca en ejercicio y el reporte de minutos en "Zona"; es decir, tiempo efectivo en que los sujetos estuvieron entre $64 \%$ y $96 \%$ de su frecuencia cardíaca máxima.

\section{Condiciones de Estudio}

Todos los sujetos se distribuyeron aleatoriamente a una de las tres condiciones del estudio: dos condiciones experimentales diseñadas de forma equivalente en volumen y tipo de actividad física, y una condición control, durante un periodo de 10 semanas, la Tabla 1 describe las características de las condiciones experimentales de estudio.

I. Programa de Educación Física Regular (PEFR): recibió 20 clases de Educación Física, dos veces por semana, 40 minutos cada vez; según el modelo regular de enseñanza. El programa incluyó: (a) ejercicios isométricos y concéntricos de fuerza con el propio peso corporal, específicos para desarrollar FET, en seguimiento de las recomendaciones de todo programa de fuerza 
pediátrico (Lloyd et al., 2014), y (b) juegos organizados de intensidad moderada y vigorosa que impliquen DFM. En doce ocasiones, se planificaron rondas de diálogo sobre mantener hábitos de vida saludable, utilizando láminas diseńadas para esta finalidad.

II. Programa de Cuentos Motores (PCM): recibió 20 Cuentos Motores diferentes, de 40 minutos cada vez, 2 veces por semana. Los Cuentos Motores se diseñaron con: (a) ejercicios isométricos y concéntricos de fuerza con el propio peso corporal, específicos para desarrollar FET, en seguimiento de las recomendaciones de todo programa de fuerza pediátrico (Lloyd et al., 2014), y (b) juegos organizados de intensidad moderada y vigorosa que impliquen DFM. En doce ocasiones, se planificaron rondas de diálogo sobre mantener hábitos de vida saludable, utilizando láminas diseñadas para esta finalidad.

III. Condición Control: Correspondiente al grupo control es decir, al grupo que no recibió ninguno de los programas descritos, de manera que los sujetos permanecieron en sus condiciones habituales y no recibieron clases de Educación Física en la institución escolar.

\section{Análisis Estadístico}

Se realizaron análisis con el Paquete Estadístico para Ciencias Sociales (SPSS Inc. Chicago, IL) versión 21. La estadística descriptiva incluyó la Media y la Desviación Estándar ( \pm DS). Se calcularon Tamaños del Efecto y se trabajó con un nivel de significancia del 95\% ( $\mathrm{p}<0.05)$.

Tabla 1. Descripción de los dos programas de estudio.

\begin{tabular}{|c|c|c|c|c|}
\hline AF de la lección, por bloque de trabajo & PCM & PEFR & $\begin{array}{l}\text { Tiempo } \\
(\mathrm{min})\end{array}$ & Sesiones \\
\hline \multirow{6}{*}{$\begin{array}{l}\text { BLOQUE DE TRABAJO A } \\
4 \text { Ejercicios de Resistencia Cardiovascu- } \\
\text { lar mediante actividades lúdicas según } \\
\text { ML }\end{array}$} & Cuidado con el canto del Viejo Sapo & \multirow{6}{*}{$\begin{array}{l}\text { Juegos organizados, } \\
\text { correspondientes al } \\
\text { desarrollo de RCV } \\
\text { con destrezas de ML }\end{array}$} & \multirow[t]{6}{*}{40} & \multirow{6}{*}{$\begin{array}{l}1^{\circ}, 2^{\circ}, 3^{\circ} \\
11^{\circ}, 12^{\circ} \mathrm{y} \\
13^{\circ}\end{array}$} \\
\hline & Piratas caza-reliquias: era prehistórica & & & \\
\hline & Misión rescate: gotas por un día & & & \\
\hline & Dos reyes y un Gobierno & & & \\
\hline & Visión nocturna, de vuelta al zoológico & & & \\
\hline & El flautista de Hamelin: cuento adaptado & & & \\
\hline \multirow{6}{*}{$\begin{array}{l}\text { BLOQUE DE TRABAJO B } \\
2 \text { Ejercicios de Fuerza } \\
2 \text { Actividades lúdicas según CO }\end{array}$} & Una riña entre comidas & \multirow{6}{*}{$\begin{array}{c}\text { Juegos organizados } \\
\text { con destrezas de CO } \\
\text { y ejercicios específicos } \\
\text { de FET }\end{array}$} & \multirow[t]{6}{*}{40} & \multirow{6}{*}{$\begin{array}{c}4^{\circ}, 5^{\circ}, 6^{\circ} \\
14^{\circ}, 15^{\circ} y \\
16^{\circ}\end{array}$} \\
\hline & La casa del Ilusionista Rafar & & & \\
\hline & La Granja & & & \\
\hline & Tío Conejo y la carreta de los quesos & & & \\
\hline & La Renuncia de la Atmósfera & & & \\
\hline & Inundación Hormiga & & & \\
\hline \multirow{6}{*}{$\begin{array}{l}\text { BLOQUE DE TRABAJO C } \\
2 \text { Ejercicios de Flexibilidad } \\
2 \text { Actividades lúdicas según CO }\end{array}$} & Una gira espacial & \multirow{6}{*}{$\begin{array}{l}\text { Juegos organizados } \\
\text { con destrezas de } \\
\text { CO y ejercicios de } \\
\text { flexibilidad }\end{array}$} & \multirow[t]{6}{*}{40} & \multirow{6}{*}{$\begin{array}{l}7^{\circ}, 8^{\circ}, 9^{\circ}, \\
17^{\circ}, 18^{\circ} y \\
19^{\circ}\end{array}$} \\
\hline & El Gigante Egoísta: cuento adaptado & & & \\
\hline & ¿Eres tú Monstruo? & & & \\
\hline & Grandes Samuráis busca brujas & & & \\
\hline & La Copa de la Vida: magia que brota del corazón & & & \\
\hline & Los niños sin hueso & & & \\
\hline \multirow{2}{*}{$\begin{array}{l}\text { BLOQUE DE TRABAJO D } \\
\text { AF, bloques A, B y C. }\end{array}$} & La venganza del Sheriff: fiestas en el Viejo Oeste & \multirow[t]{2}{*}{ AF por estaciones } & \multirow[t]{2}{*}{40} & \multirow[t]{2}{*}{$10^{\circ}$ y $20^{\circ}$} \\
\hline & Olimpiadas de los Juegos Extremos & & & \\
\hline
\end{tabular}

\section{Discusión de los resultados}

Se reclutaron 67 escolares que no contaban con clases de Educación Física, pero solo 55 completaron el estudio, con una edad promedio de $9.36 \pm 0.71$ ańos, distribuidos aleatoriamente en tres grupos PEFR ( $n=16)$, PCM ( $n=18)$ y Grupo Control (GC) $(n=21)$. Se excluyeron en los análisis, los re- sultados de los sujetos con: (1) restricción médica para hacer AFMV, (2) padecimientos crónicos limitantes del desarrollo motor grueso, y (3) ausentismo de más de 4 sesiones de intervención.

Todos los sujetos mostraron tener un peso corporal, talla e IMC saludable (ver Tabla 2) según directrices de la Organización Mundial de la Salud (OMS, 2016) que indican que un 
niño y niña de 9 a 10 años, debe tener un $\mathrm{IMC}=17.5$ a 23.5 $\left(\mathrm{kg} / \mathrm{m}^{2}\right)$, talla $=130$ a $140(\mathrm{~cm})$, y peso $=30.00$ a $35.00(\mathrm{~kg})$. Hubo homogeneidad en características antropométricas y la distribución de la cantidad de niños y niñas, en cada uno de los grupos.

Tabla 2. Características generales (Media +DS) de los sujetos participantes.

\begin{tabular}{|c|c|c|c|c|c|}
\hline & GC $(n=21)$ & $\operatorname{PEFR}(\mathrm{n}=23)$ & PCM (n=23) & $\mathrm{F}$ & $\mathrm{p}$ \\
\hline Edad (años) & $9.43+0.70$ & $9.32+0.69$ & $9.33+0.74$ & 0.16 & 0.852 \\
\hline Talla $(\mathrm{cm})$ & $1.35+5.72$ & $1.34+7.83$ & $1.34+6.32$ & 0.17 & 0.839 \\
\hline Peso $(\mathrm{kg})$ & $31.30+6.06$ & $31.22+9.21$ & $32.67+10.41$ & 0.19 & 0.827 \\
\hline \multirow[t]{2}{*}{ IMC $\left(\mathrm{kg} / \mathrm{m}^{2}\right)$} & $17.19+2.63$ & $17.19+3.00$ & $18.12+4.31$ & 0.53 & 0.588 \\
\hline & & & & $\mathrm{Chi}^{2}$ & $\mathrm{p}$ \\
\hline Sexo & $q=9 ; \hat{\partial}=12$ & $q=11 ; \hat{\jmath}=12$ & $q=12 ; \hat{\partial}=11$ & 0.38 & 0.826 \\
\hline
\end{tabular}

Nota: GC=Condición control; PEFR= Programa Educación Física Regular; PCM= Programa Cuentos Motores; +DS=desviación estándar; $q=$ mujeres; $\partial$ =hombres; F= análisis de Anova 1 vía para grupos independientes; $\mathbf{C h i}^{2}=$ análisis de comparación no paramétrico. Significancia de $\mathrm{p}<0.05$

Análisis complementarios indicaron ausencia de diferencias significativas ( $p>0.05$ ) en todas las variables dependientes al inicio del estudio. Asimismo, por sexo según la medición y grupo de estudio (en RCV F=0.83; $\mathrm{p}=0.438$; en FET $\mathrm{F}=0.02$; $\mathrm{p}=0.977$; en $\mathrm{ML} \mathrm{F}=1.02 ; \mathrm{p}=0.365$; en $\mathrm{CO} F=0.36 ; \mathrm{p}=0.693$ y en CDMG F=0.78; p=0.464). Posiblemente porque los sujetos iniciaron en las mismas condiciones y no habían recibido Educación Física en la institución escolar; por lo tanto cualesquiera que fueran los estímulos de actividad física que hubieran tenido dentro y fuera del contexto escolar fue una constante que no influyó en los resultados por sexo. Además, el promedio de edad indicó niñez (ausencia de características sexuales secundarias) considerando que las diferencias por sexo en aptitud física y desarrollo motor grueso principal-

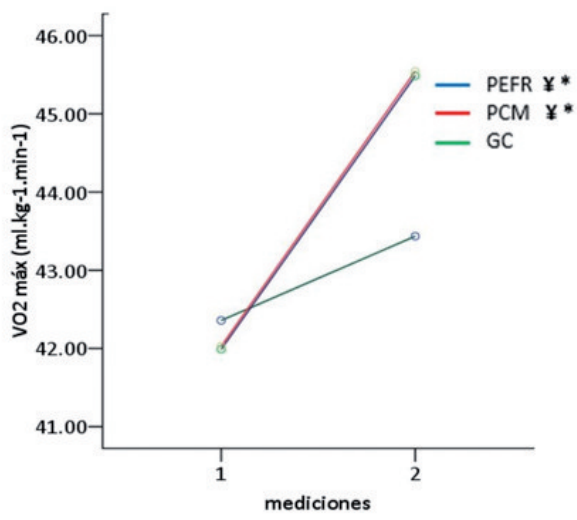

Figura 1. Efecto de PEFR y PCM sobre la RCV. ${ }^{*}=p<0.05$ diferencia entre pre-test $y$ post-test del respectivo grupo; $¥=p<0.05$ respecto al GC.

Según la literatura científica (Wilmore y Costill, 2007; Lloyd et al.,2014) mientras que las adaptaciones fisiológicas al entrenamiento de RCV y fuerza muscular en los adultos tiende a ser más específica y rigurosa a sus demandas metabólicas, en los niños y niñas funciona diferente puesto que cursan mente se encuentran cuando se presenta la adolescencia (Wilmore \& Costill, 2007; Gallahue, Ozmun \& Goodway, 2012).

Mediante el análisis de ANOVA se encontraron interacciones dobles significativas en la $\operatorname{RCV}(\mathrm{F}=3.80, \mathrm{p}=0.028)$ y la FET ( F=6.65, p=0.002), ver Figuras 1 y 2, y Tabla 3. Se determinó que los sujetos intervenidos obtuvieron mejoría en la RCV y FET de pre-test a post-test $(\mathrm{p}<0.05)$ y no así el grupo control $(p>0.05)$. Entre los grupos intervenidos no hubo diferencias significativas $(\mathrm{p}>0.05)$ en los resultados del post-test, lo que indica que los programas fueron similarmente eficaces para la mejora de estos componentes. Estos resultados son consistentes con otras investigaciones (Faigenbeaum et al., 2011; Eather et al., 2013; Allen, Hannon, Burns, \& Williams, 2014).

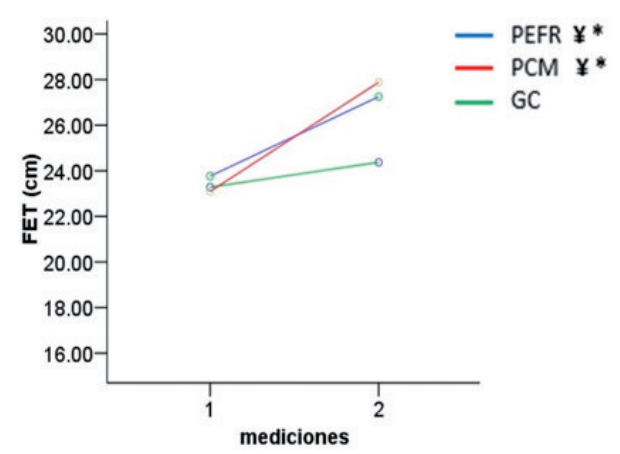

Figura 2. Efecto de PEFR y PCM sobre la FET. * ${ }^{*}=p<0.05$ diferencia entre pre-test y post-test del respectivo grupo; $¥=p<0.05$ respecto al $G C$.

por un período de crecimiento y desarrollo "ventana de oportunidad” lo que aunado a estímulos dirigidos, sostenidos y específicos para el desarrollo de RCV y FET y considerando recomendaciones de todo programa pediátrico, aumenta: (1) cambios hematológicos sobre el crecimiento de volumen san- 
guíneo principalmente en plasma, hematocrito y hemoglobina, que justifica la mejora significativa $(\mathrm{p}=0.028)$ en la RCV de los niños intervenidos; (2) cambios neurológicos en mejora de la técnica de la coordinación motora, aumento de la activación motora y mecanismos fisiológicos que intervienen en el cambio de la fuerza muscular en la niñez, principalmente mediante hipertrofia de fibras musculares individuales a través de incrementos de miofilamentos y miofibrillas en las fibras existentes, que justifica la mejora $(\mathrm{p}=0.002)$ en la FET de los sujetos intervenidos.

El rango de los valores de Tamaño de Efecto (TE) de PEFR y PCM fueron mayores respecto al 0, no así los TE del GC. El grupo que recibió PCM mostró TE más grandes en RCV y FET (TE=1.39 y TE=1.36; respectivamente). El grupo que recibió el $\mathrm{PEFR}$ obtuvo $\mathrm{TE}=0.98$ y $\mathrm{TE}=0.71$; respectivamente). Es muy probable que los sujetos que recibieron el PCM mantuvieron en mayor constancia del esfuerzo físico a través de los Cuentos Motores, ya que esta metodología demanda una atención constante y diferentes esfuerzos físicos consecutivos hasta finalizar el cuento. El grupo control obtuvo $\mathrm{TE}$ pequeños ( $\mathrm{TE}=0.32$ y $\mathrm{TE}=0.12$; respectivamente).

En términos de salud los valores de RCV (> $40.2 \mathrm{ml} \cdot \mathrm{kg}^{-}$ $\left.{ }^{1} \cdot \mathrm{min}^{-1}\right)$ y FET $(15.24$ a $30.48 \mathrm{~cm}$ ) están dentro del rango de aptitud física saludable acorde a la edad de 9 a 10 años, según directrices aprobadas del FITNESSGRAM ${ }^{\circledR}$ (2014), lo que permite afirmar que los programas fueron eficaces para promover bienestar en tanto que les permitió desarrollar mayor RCV y FET, en comparación a lo que les pudo haber proporcionado el juego libre.

Asimismo, la Tabla 4 describe los resultados de las interacciones dobles significativas en $\mathrm{ML}(\mathrm{F}=8.22, \mathrm{p}=0.0001)$, $\mathrm{CO}(\mathrm{F}=9.70, \mathrm{p}=0.0001)$ y por ende en el CDMG $(\mathrm{F}=25.79$, $\mathrm{p}=0.0001$; ver Figura 3). Los sujetos intervenidos obtuvieron mejoría en casi todas las DFM $(\mathrm{p}<0.05)$, el grupo control no mejoró en ninguna de las DFM. Entre los grupos intervenidos no hubo diferencias significativas $(\mathrm{p}>0.05)$ en los resultados del post-test, lo que indica que los programas fueron similarmente eficaces para la mejora de estos componentes. Estos resultados son consistentes con otros estudios (JiménezDíaz \& Araya-Vargas, 2009; Logan et al., 2015) sobre la efi- cacia de intervenciones motrices específicas para la mejora de las DFM, lo cual no se alcanza sin la instrucción, la práctica dirigida y el reforzamiento sostenido a través del tiempo.

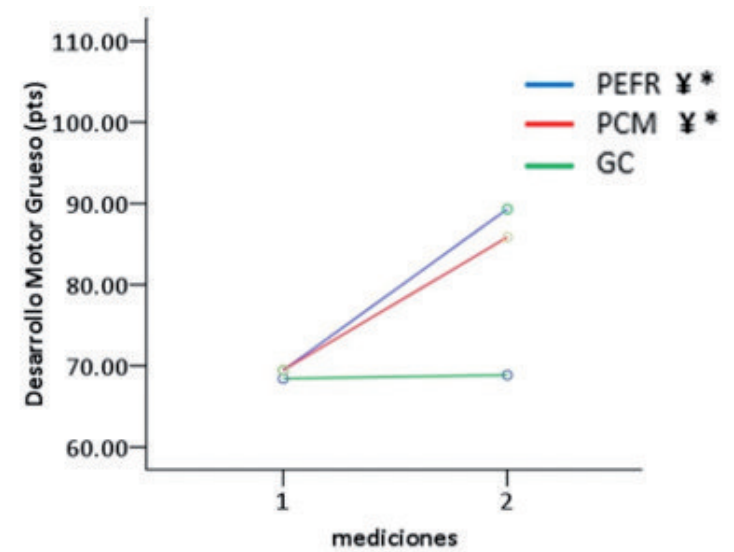

Figura 3. Efecto de PEFR y PCM sobre el CDMG. * $=p<0.05$ diferencia entre pre-test y post-test del respectivo grupo; $¥=p<0.05$ respecto al GC.

En los dos grupos intervenidos PEFR y PCM, se encontró $\mathrm{TE}$ grandes (superiores a 0 ) en CDMG ( $\mathrm{TE}=1.86$ y $\mathrm{TE}=1.38$; respectivamente). El grupo control mostró TE pequeño (TE=0.04).

Se calcularon los TE de cada una de las DFM analizadas en los grupos intervenidos. El grupo que recibió el PCM obtuvo mayores TE en carrera $=0.72$; galope $=1.81$; lanzamiento sobre el hombro=1.23; lanzamiento debajo del hombro=1.75 y patear=0.10. Por su parte, el grupo que recibió el PEFR obtuvo $\mathrm{TE}$ en carrera $=0.62$; galope $=0.55$; lanzamiento sobre el hombro=0.39; lanzamiento debajo del hombro=0.98 y patear=0.07. Eventualmente la escenografía, materiales didácticos utilizados por los sujetos para representar los personajes del cuento y la música, fueron factores motivacionales importantes en la ejecución de las acciones motoras de forma más eficiente, que pudo favorecer a nivel intrínseco, mayores ajustes en la coordinación motora en carrera, galope y lanzamientos. Es bien sabido que los factores motivacionales influyen sobre el rendimiento motor grueso en los niños y niñas (Lakusić, 2015).

Tabla 3. Anova mixto de medidas repetidas en un factor para las variables de aptitud física (Los valores se presentan como la Media $+\mathrm{DS}$ )

\begin{tabular}{|c|c|c|c|c|c|c|c|}
\hline & \multicolumn{2}{|c|}{$\mathrm{GC}(\mathrm{n}=21)$} & \multicolumn{2}{|c|}{$\operatorname{PEFR}(\mathrm{n}=16)$} & \multicolumn{2}{|c|}{ PCM $(\mathrm{n}=18)$} & \multirow{2}{*}{$\begin{array}{c}\mathrm{p}<0.05 \\
\text { (Interacción) }\end{array}$} \\
\hline & Pre-test & Post-test & Pre-test & Post-test & Pre-test & Post-test & \\
\hline $\mathrm{RCV}\left(\mathrm{ml} \cdot \mathrm{kg}^{-1} \cdot \mathrm{min}^{-1}\right)$ & $42.35+2.86$ & $43.43+3.80$ & $41.99+2.75$ & $45.48+4.20 * ¥$ & $42.02+1.71$ & $45.54+3.12^{*} ¥$ & 0.028 \\
\hline FET (pulg) & $23.26+3.29$ & $24.35+1.22$ & $23.85+1.87$ & $26.85+1.42 * ¥$ & $23.08+1.30$ & $27.69+1.36^{*} ¥$ & 0.002 \\
\hline
\end{tabular}

Nota: GC=Condición control; PEFR= Programa Educación Física Regular; PCM= Programa Cuentos Motores; RCV= Resistencia Cardiovascular; FET= Fuerza Extensora de Tronco; $\mathrm{p}=$ significancia de interacción. Post Hoc de Efectos Simples $(\mathrm{p}<0.05):{ }^{*}=$ diferencias entre pre-test y post-test; $¥=$ diferencias respecto grupo control.

Contrariamente, el grupo que recibió el PEFR obtuvo mayores $\mathrm{TE}$ en saltar $=1.44$; rebotar $=0.98$; batear $=0.83 \mathrm{y}$ apañar=1.12. Dado que, el grupo que recibió el PCM alcanzó $\mathrm{TE}$ en saltar $=0.45$; rebotar $=0.39$; batear $=0.36$ y apañar $=0.76$. 
Naturalmente, cada DFM posee un grado de complejidad, resultando más sencillo correr y galopar que desplazarse lateralmente, más aún mediante zancadas y saltos horizontales. Además, las destrezas de CO son mucho más complejas que las destrezas de ML porque demandan mayor grado de coordinación motora; ahora bien, las destrezas de batear, rebotar y apañar resultan más complejas que las destrezas de lanzamiento (Gallahue, Ozmun \& Goodway, 2012). Entre mayor sea la complejidad de la destreza, mayor es el tiempo requerido dedicado a la práctica, corrección y reforzamiento de la destreza, para lograr su ejecución en una etapa madura (Logan et al., 2015).

Considerando la naturaleza de los Cuentos Motores, no es conveniente admitir constantes interrupciones para corregir y reforzar la ejecución de las destrezas durante el desarrollo del cuento, de lo contrario disminuiría la calidad de la actividad en sí misma. En cambio, la metodología del PEFR si admite el cese requerido para la corrección y reforzamiento sin afectar por ello la naturaleza de las actividades propuestas; por lo tanto, proporciona mayor oportunidad de aprendizaje de las DFM más complejas.

El brinco, zancada, patear y batear no cambiaron significativamente ( $p>0.05)$ en ningún de los grupos de estudio. La zancada y batear son destrezas inusuales en los niños y niñas costarricenses, tal vez un tiempo de práctica mayor hubiese generado los cambios esperados. La zancada es un movimiento que se ejecuta durante la carrera y suele ser practicado como ejercicio de progresión de velocidad por los atletas. El bateo se práctica muy poco, al menos en las instituciones escolares públicas costarricenses, en parte por falta del material didáctico y el poco auge (aunque va en aumento) que tienen el béisbol y el softball en este país. En cuanto a brincar y patear, todos los sujetos puntuaron muy cerca de la calificación máxima desde el inicio del estudio, y por lo tanto no se reflejaron cambios relevantes en estas destrezas.

Según los criterios del TGMD- ${ }^{\circledR}$, un CDMG aceptable para los sujetos de estudio (valor de desarrollo promedio esperado en consideración a la edad y sexo del individuo) debe puntuar dentro del rango 90 a 110 del valor estandarizado CDMG. En la medición inicial, todos los sujetos mostraron valores clasificados en la escala: CDMG muy pobre; al puntuar $<70$, que indica que los sujetos iniciaron con un desarrollo motor grueso esperado en niños de 4 a 6 ańos de edad. A finalizar el estudio, los sujetos intervenidos no lograron puntuar dentro del rango aceptable esperado, contrariamente alcanzaron valores en LM esperados en niños y nińas de 5 a 6 años, y valores en $\mathrm{CO}$ esperados en niños y niñas de 8 a 9 años.

Tabla 4. Anova mixto de medidas repetidas en un factor para las variables de DFM (Se presentan en puntajes crudos como la Media \pm DS).

\begin{tabular}{|c|c|c|c|c|c|c|c|}
\hline & \multicolumn{2}{|c|}{ GC (n=21) } & \multicolumn{2}{|c|}{$\operatorname{PEFR}(\mathrm{n}=16)$} & \multicolumn{2}{|c|}{ PCM (n=18) } & \multirow[t]{2}{*}{$\begin{array}{c}\mathrm{p}<0.05 \\
\text { (Interacción) }\end{array}$} \\
\hline & Pre-test & Post-test & Pre-test & Post-test & Pre-test & Post-test & \\
\hline ML & $28.28+3.73$ & $28.00+4.69$ & $29.25+6.27$ & $37.18+5.15^{*} ¥$ & $29.88+3.42$ & $35.38+7.03^{*} ¥$ & 0.000 \\
\hline $\mathrm{CO}$ & $34.95+6.63$ & $35.66+6.39$ & $34.43+4.54$ & $41.75+3.80^{*} ¥$ & $33.83+6.81$ & $40.55+4.82 * ¥$ & 0.000 \\
\hline $\begin{array}{l}\text { CDMG } \\
\text { (puntaje estandarizado) }\end{array}$ & $68.42 \pm 10.24$ & $68.85 \pm 9.31$ & $69.73 \pm 8.76$ & $89.31 \pm 11.99^{*} ¥$ & $69.59 \pm 8.79$ & $85.83 \pm 14.06^{*} ¥$ & 0.000 \\
\hline Carrera & $7.04+1.28$ & $6.85+1.44$ & $7.31+1.19$ & $8.00+1.01 ¥$ & $6.22+1.66$ & $7.22+1.00 *$ & 0.031 \\
\hline Galope & $4.09+1.48$ & $4.19+1.24$ & $5.00+1.93$ & $6.12+2.12 * ¥$ & $4.05+1.51$ & $6.66+1.37 * ¥$ & 0.001 \\
\hline Deslizamiento Lateral & $6.47+1.50$ & $6.04+1.71$ & $6.06+2.54$ & $7.62+1.08 * ¥$ & $6.55+1.46$ & $7.05+1.39$ & 0.020 \\
\hline Zancada & $2.23+2.93$ & $2.33+2.15$ & $2.12+1.78$ & $2.31+2.96$ & $2.22+2.04$ & $2.44+2.83$ & 0.501 \\
\hline Brinco & $6.33+2.00$ & $7.19+2.22$ & $7.93+2.08$ & $8.56+1.59$ & $8.55+1.82$ & $8.72+1.40$ & 0.647 \\
\hline Salto & $2.09+1.92$ & $1.38+1.88$ & $1.81+2.04$ & $4.56+1.75 * ¥$ & $2.27+1.67$ & $3.27+2.61 ¥$ & 0.001 \\
\hline Rebotar & $5.42+2.27$ & $5.14+2.22$ & $4.81+2.22$ & $6.68+1.49 * ¥$ & $5.72+1.93$ & $6.44+1.75 ¥$ & 0.009 \\
\hline Apañar & $5.80+0.51$ & $4.66+1.01^{*}$ & $5.31+0.87$ & $6.00+0.01^{*} ¥$ & $5.50+0.78$ & $5.94+0.23 ¥$ & 0.000 \\
\hline Lanzar sobre hombro & $4.47+2.08$ & $6.85+2.05^{*}$ & $7.37+1.20$ & $7.75+0.68$ & $5.22+2.86$ & $7.77+0.64^{*}$ & 0.013 \\
\hline Lanzar debajo del hombro & $5.14+2.24$ & $4.90+2.23$ & $4.25+1.43$ & $6.00+2.06^{*} ¥$ & $3.44+1.14$ & $5.77+1.51^{*} ¥$ & 0.000 \\
\hline Patear & $6.85+1.36$ & $6.98+1.45$ & $6.50+1.54$ & $6.62+1.74$ & $6.33+1.84$ & $6.50+1.29$ & 0.954 \\
\hline Batear & $7.23+2.04$ & $7.34+2.19$ & $7.18+2.37$ & $8.81+1.42$ & $7.61+2.30$ & $8.38+1.85$ & 0.076 \\
\hline
\end{tabular}

Nota: GC=Condición control; PEFR= Programa Educación Física Regular; PCM=Programa Cuentos Motores; ML= Movimientos de Locomoción; CO= Control de Obje-
tos; $\mathrm{p}=$ significancia de interacción. Post Hoc de Efectos Simples $(\mathrm{p}<0.05):{ }^{*}=$ diferencias entre pre-test $\mathrm{y}$ post-test; $¥=$ diferencias respecto al grupo control. 
Sin embargo, el PEFR y el PCM fueron útiles para que la competencia motora de los sujetos mejorara significativamente ( $\mathrm{p}=0.001)$, pasando de un valor de CDMG muy pobre $(69.73 \pm 8.76$ y $69.50 \pm 8.79$, respectivamente) a un valor de CDMG bajo según la media esperada $(89.31 \pm 11.99$ y $85.83 \pm 14.06$, respectivamente). Un tiempo mayor de intervención hubiese permitido alcanzar el valor de CDMG óptimo. Por su parte, el grupo control mantuvo valores de CDMG muy pobre $(68.42 \pm 10.24$ a $68.85 \pm 9.31)$ a lo largo del estudio. Resulta imprescindible que los niños y niñas reciban Educación Física en la institución educativa, ya que su ausencia priva el alcance de DFM en su etapa madura, por ende afecta negativamente el desarrollo motor grueso de los niños y niñas, y limita sus posibilidades para participar en diferentes actividades físicas a corto, mediano y largo plazo (Benjumea, Alfonso, \& Ramírez, 2015).

Finalmente, la cantidad de minutos efectivos de AFMV en ambos programas $(\mathrm{PEFR}=29.15 \pm 8.74$ y $\mathrm{PCM}=31.35 \pm 8.20$ ) fue similar ( $\mathrm{p}=0.497$, según análisis de prueba $\mathrm{t}$-Student) y superior al 50\% del tiempo total de una lección, lo que indica que los dos programas fueron equivalentes entre sí, y lograron cumplir con las demandas del ACSM, (2018) en promoción de la salud escolar.

\section{Conclusión}

Las estrategias de los programas fueron similarmente eficaces para ofrecer AFMV y mejorar RCV, FET y CDMG de los nińos y nińas participantes que no contaban con clases de Educación Física en la institución educativa. Este estudio fue novedoso porque demostró la eficacia de un Programa de Cuentos Motores sobre componentes de aptitud física y desarrollo motor grueso en niños y niñas escolares, que anteriormente no había sido estudiado.

Futuras investigaciones deben continuar estudiando estrategias interdisciplinarias de Educación Física y Cuentos Motores, en otras variables que atañen al manejo de la Obesidad Infantil y la promoción de la salud escolar.

\section{Referencias}

1. ACSM/American Collage of Sport Medicine (2018) ACSM's guidelines for exercise testing and prescription (10th ed.) Philadelphia, PA: Wolters Kluwer Health.

2. Allen, B. A., Hannon, J. C., Burns, R. D. \& Williams, S. M. (2014). Effect of a core conditioning intervention on tests of trunk muscular endurance in school-aged children. The Journal of Strength \& Conditioning Research, 28(7), 2063-2070.

3. Arce León, A. P. \& Claramunt Garro, M. (2009). Educación para la Salud como tema transversal en el sistema educativo costarricense. Guia para docentes y personal de salud. (2da ed.). Costa Rica: Ministerio de Educación Pública.

4. Aparicio Sacristán, J. (2014). El desarrollo de las competencias básicas en Educación Física. (Tesis de maestría, Universidad de Valladolid). Recuperado de http://uvadoc.uva.es/handle/10324/5002

5. Benjumea, J. M. C., Afonso, J. R. \& Ramírez, J. M. (2015). Assessment of motor coordination in students aged 6 to 11 years. Journal of Physical Education and Sport, 15(4), $765-774$.

6. Eather, N., Morgan, P. J. \& Lubans, D. R. (2013). Improving the fitness and physical activity levels of primary school children: Results of the Fit-4-Fun group randomized controlled trial. Preventive medicine, 56(1), 12-19.

7. Escriche, C. C., Muñoz, S. R., Sos, A. A., \& Casterad, J. Z. (2018). Cumplimiento de las recomendaciones de práctica de actividad física en función de los cutoffs points y el género en estudiantes universitarios españoles. Sportk: revista euroamericana de ciencias del deporte, 7(1), 9-18.

8. Faigenbaum, A. D., Farrell, A., Fabiano, M., Radler, T., Naclerio Ayllón, F. J., Ratamess, N. A., \& Myer, G. D. (2011). Effects of integrative neuromuscular training on fitness performance in children. Pediatric Exercise Science, 23, 573-584.

9. Gallahue, G. L., Ozmun, J. C., \& Goodway, J. D. (2012). Development of Fundamental Movement: Manipulation Skills. In Gallahue, G. L., Ozmun, J. C., \& Goodway, J. D. (Eds.), Understanding Motor Development: infants, children, adolescents, adults -7th ed. (str. 191-203), New York, McGraw-Hill

10. García, G.C. \& Secchi, J. D. (2014). Test Course Navette de 20 metros con etapas de un minuto. Una idea original que perdura hace 30 años. Apunts Med Esport, 49(183), 93-103.

11. Herrera, E. M. \& Barbero, M. d. S. M. (2013). El cuerpo, la expresión y el medio acuático. Una experiencia integradora. Retos: nuevas tendencias en educación física, deporte y recreación, 24, 176-183.

12. Jiménez, J. \& Araya, G. (2009). Efecto de una intervención motriz en el desarrollo motor, rendimiento académico y creatividad en preescolares. Pensar en Movimiento. Revista de Ciencias del Ejercicio y la Salud. 7(1), 11-22.

13. Lloyd, R. S., Faigenbaum, A. D., Stone, M. H., Oliver, J. L., Jeffreys, I., Moody, J., . . \& \& Howard, R. (2014). Posicionamiento sobre el entrenamiento de Fuerza en jóvenes. Consenso Internacional de 2014. Archivos de medicina del deporte. Revista de la Federación Española de Medicina del Deporte y de la Confederación Iberoamericana de Medicina del Deporte, 160, 111-124.

14. Logan, S. W., Webster, E. K., Getchell, N., Pfeiffer, K. A. \& Robinson, L. E. (2015). Relationship Between Fundamental Motor Skill Competence and Physical Activity During Childhood and Adolescence: $A$ Systematic Review. Kinesiology Review, 4(4), 416-426.

15. Ministerio de Salud. (2009). Encuesta Nacional de Nutrición, Costa Rica. San José, Costa Rica: Recuperado de http://www.ministeriodesalud.go.cr/.../33-encuesta-nacional-de-nutricion

16. Morgan, P. J., Barnett, L. M., Cliff, D. P., Okely, A. D., Scott, H. A., Cohen, K. E. \& Lubans, D. R. (2013). Fundamental movement skill interventions in youth: A systematic review and meta-analysis. Pediatrics, 135(5), 1361-1383.

17. Organización Mundial de la Salud-OMS. (2016). Datos sobre la Obesidad. Recuperado de http://www.who.int/features/factfiles/obesity/facts/es/.

18. Reyes Alonso, V. (2013). Actividades físicas en el medio natural y Educación Fisica. (Tesis de maestría, Universidad de Valladolid). Recuperado de http://uvadoc.uva.es/handle/10324/3225

19. Sbruzzi, G., Eibel, B., Barbiero, S. M., Petkowicz, R. O., Ribeiro, R. A., Cesa, C. C., . . \& \& Souza, W. B. (2013). Educational interventions in childhood obesity: A systematic review with meta-analysis of randomized clinical trials. Preventive medicine, 56(5), 254-264.

20. Wilmore, J. \& Costill, D. (2007). Fisiología del Esfuerzo y del Deporte. España: Paidotribo. 